\title{
Simulation of imprinted emulsion prepolymerization mixtures
}

\author{
Gustaf D Olsson $^{1}$, Klaus Niedergall ${ }^{2}$, Monika Bach ${ }^{2,3}$, Björn CG Karlsson ${ }^{1}$, Günter Tovar ${ }^{2,3}$ and \\ Ian A Nicholls ${ }^{1,4}$
}

Polymer Journal (2015) 47, 827-830; doi:10.1038/pj.2015.63; published online 30 September 2015

The aim of this study was to develop protocols for and evaluate the use of all-atom full system molecular dynamic (MD) simulations of emulsion systems in the development of molecularly imprinted polymers (MIPs). Here, we report on the first, to the best of our knowledge, use of all-component MD studies to simulate and evaluate MIP miniemulsion prepolymerization mixtures; in this case, the mixtures used in the synthesis of a series of MIP-nanoparticles (MIP-NPs). The insights gained were correlated to the colloidal stability and NP physical characteristics (morphology, size) and to the template rebinding capacity.

The studied materials consisted of a series of MIP-NPs selective for the endocrine disruptor bisphenol A (BPA). ${ }^{1}$ The widespread use of BPA, particularly in polymer production, ${ }^{2}$ and its potential adverse health effects have increased the concerns regarding human exposure, which has led to a demand for the development of improved methods for BPA detection and removal. The molecular imprinting technique, ${ }^{3-5}$ which provides access to polymeric materials with predetermined ligand selectivity, is potentially such a method. The concept is based on the entrapment of the template-functional monomer complexes that are present in the prepolymerization phase through the polymerization process. Experimental strategies have been a central aspect of molecularly imprinted polymer (MIP) development. ${ }^{6}$ Recently, the all-component modeling of prepolymerization mixtures has demonstrated high value for both MIP design and polymer performance assessment. ${ }^{7-10}$ The composition of polymerization mixtures (monomers, solvent(s), stoichiometries) and procedure influence both the physical characteristics and the recognition properties of the final material. Polymer nanoparticles can be obtained by miniemulsion polymerization. ${ }^{11-13}$ MIP-NPs have recently received attention because of their potential use in biotechnological and medical applications and in miniaturized lab-on-a-chip systems. ${ }^{14-17}$ The inclusion of a dispersion phase increases the complexity of the system because the resulting phase boundaries permit partitioning effects, which in turn influence the relative concentrations of and interactions between the prepolymerization mixture components. This added complexity makes the prediction of the outcome of polymerization reactions performed in emulsions more difficult. ${ }^{18}$ The situation is even more complex in the case of molecular imprinting using emulsion polymerization, where the presence of the template introduces an additional ensemble of equilibria. We envisioned that the development of a method for the modeling of emulsion polymerization systems would therefore be of general interest, and we applied this approach to the study and optimization of molecularly imprinted materials selective for BPA.

The MD simulation of five miniemulsion prepolymerization mixtures was undertaken, as shown in Table 1 . The systems consisted of BPA, methacrylic acid (MAA), ethylene glycol dimethacrylate (EGDMA), hexadecane, dimethoxyphenylacetophenone (DMP), sodium dodecyl sulfate (SDS), hexadecane (HDE) and water, either in the presence, $\mathrm{m}$ (MIP1) and $\mathrm{m}$ (MIP2), or absence, $\mathrm{m}$ (REF), of BPA. Two further systems with methacrylate and oxonium $\left(\mathrm{H}_{3} \mathrm{O}^{+}\right)$ions were also simulated, $\mathrm{m}(\mathrm{DP} 1)$ and $\mathrm{m}(\mathrm{DP} 2)$.

See the Supplementary Table S1 for full details of system composition, Figure 1 for partial structural information and Supplementary Figure S1 for detailed structural information. All systems maintained

Table 1 Compositions of modeled systems

\begin{tabular}{lccccc}
\hline Molecule $^{\mathrm{a}}$ & $m($ REF $)$ & $m(M I P 1)$ & $m(M I P 2)$ & $m(D P 1)$ & $m(D P 2)$ \\
\hline BPA & - & 100 & 25 & 100 & 25 \\
MAA & 100 & 100 & 100 & 50 & 50 \\
MAD & - & - & - & 50 & 50 \\
Water & 4088 & 4088 & 4088 & 4038 & 4038 \\
H30 & - & - & - & 50 & 50 \\
\hline
\end{tabular}

Abbreviations: BPA, bisphenol A; DP, including deprotonated MAA (MAD); EGDMA, ethylene glycol dimethacrylate; HDE, hexadecane; H3O, oxonium; MAA, methacrylic acid; MAD, methacrylate; MIP, molecularly imprinted polymer; REF, reference; SDS, sodium dodecyl sulfate. ${ }^{a}$ Abbreviations of molecules used in simulations and the number of molecules included in each aAbbreviations of molecules used in simulations and the number of molecules included in each
system. The number of EGDMA, HDE, DMP and SDS molecules was kept constant at 400, 16, 5 and 4 , respectively, in all systems modeled. All amounts were representative of experimental systems. See Supplementary Table S1 for detailed information regarding compositions and experimental compositions.

\footnotetext{
${ }^{1}$ Linnaeus Centre for Biomaterials Chemistry, Linnaeus University, Kalmar, Sweden; ${ }^{2}$ Fraunhofer Institute for Interfacial Engineering and Biotechnology IGB, Stuttgart, Germany; ${ }^{3}$ Stuttgart University, Institute for Interfacial Engineering and Plasmatechnology IGVT, Stuttgart, Germany and ${ }^{4}$ Department of Chemistry-BMC Uppsala University, Uppsala, Sweden

Correspondence: Professor IA Nicholls, Linnaeus Centre for Biomaterials Chemistry, Linnaeus University, Norra vägen 49, Kalmar 39182, Sweden.

E-mail: ian.nicholls@Inu.se
}

Received 12 December 2014; revised 16 June 2015; accepted 24 June 2015; published online 30 September 2015 
phase separation throughout the simulations without the use of restraints, which indicated that the systems modeled represent stable emulsions (Supplementary Figure S2).

The results from hydrogen bond analyses revealed that the predominant interaction of BPA (on average, 90\% of the simulation time per template) was with EGDMA (Table 2). Closer examination revealed this complex to be well defined (Supplementary Figures S2 and S3), which indicates fortuitous good complementarity between these structures. The degree of EGDMA-BPA complex formation did not decrease with increasing amounts of BPA (Table 2), thus indicating that the organic phase was not saturated with respect to the template. Furthermore, although the MAA:BPA stoichiometry was

Table 2 Hydrogen bond interactions with BPA

\begin{tabular}{lrr}
\hline Molecule $^{\mathrm{a}}$ & $m(M I P 1)(\%)^{\mathrm{b}}$ & $m(M I P 2)(\%)^{\mathrm{b}}$ \\
\hline MAA & 13.3 & 13.6 \\
EGDMA & 90.5 & 87.0 \\
BPA & 3.6 & 3.9 \\
Water & 42.5 & 41.5 \\
SDSc & 0.0 & 0.6 \\
\hline
\end{tabular}

Abbreviations: BPA, bisphenol A; EGDMA, ethylene glycol dimethacrylate; MAA, methacrylic acid; MAD, methacrylate; MIP, molecularly imprinted polymer; SDS, sodium dodecyl sulfate. acid, MAD, methacylate; MIP,

bThe sums of average percent (per BPA molecule) of the total simulation time ( $5 \mathrm{~ns}$ ) (see Supplementary Table S5-S8 for details).

cOnly the dodecyl sulfate chain was subjected to evaluation. four times higher in m(MIP2), only minor differences were observed in the radial distribution function (Supplementary Figure S5) and hydrogen bond analysis data on the interaction of these structures (Table 2). The reason for this effect is most likely the high concentration of EGDMA relative to BPA in the mixtures, which saturated these interaction points through the electrostatic interaction of negatively charged accessible oxygen atoms of the crosslinker.

In contrast to the EGDMA-BPA interactions, BPA displayed only moderate contact with the functional monomer (MAA) and other prepolymerization mixture components (Table 2, Supplementary Figures S4 and S5, Supplementary Table S2 and Supplementary Tables S3-S8). This result indicates, based on the observed differences in the extent of interaction between the monomers and template, that rebinding should be ascribed to sites arising predominantly from BPA-EGDMA complexes, and not from MAA-BPA interactions. The stabilities of the template-monomer hydrogen bond interactions were very similar when comparing the crosslinker- and functional monomer-BPA hydrogen bond interactions of comparable hydrogen bond acceptors of monomers (Supplementary Tables S4). This result implies that the extent of hydrogen bonding is dependent on both the physicochemical properties of the different monomers and their relative stoichiometries.

An additional aspect is MAA's weak acidic nature. In the organic phase, the monomer would not be ionized to any significant extent. However, upon coming into contact with the aqueous phase, this event is anticipated and results in a net flux of MAA into the aqueous a<smiles>CC(C)(c1ccc(O)cc1)c1ccc(O)cc1</smiles>

b<smiles>C=C(C)C(=O)O</smiles>

C<smiles>C=C(C)C(=O)OCCOC(=O)C(=C)C</smiles>

Figure 1 Structures of (a) the template, bisphenol A (BPA), and the polymerizable monomers, (b) methacrylic acid (MAA) and (c) ethylene glycol dimethacrylate (EGDMA). For further structural information, see Supplementary Figure S1.

Table 3 Polymer physical properties

\begin{tabular}{|c|c|c|c|c|}
\hline & $(R E F 2)^{a}$ & $(M I P 2)^{A}$ & $(R E F 3)^{b}$ & $(M I P 3)^{b}$ \\
\hline Polydispersity index & $0.183 \pm 0.062$ & $0.151 \pm 0.022$ & $0.342 \pm 0.071$ & $0.357 \pm 0.064$ \\
\hline BET surface area $\left(m^{2} g^{-1}\right)$ & $53.1 \pm 1.9$ & $38.1 \pm 2.8$ & $33.9 \pm 1.1$ & $30.1 \pm 2.1$ \\
\hline
\end{tabular}

Abbreviations: BET, Brunauer-Emmett-Teller; EGDMA, ethylene glycol dimethacrylate; MAA, methacrylic acid; MAD, methacrylate; MIP, molecularly imprinted polymer; REF, reference.

aColumns contain data from the poly-MAA-co-EGDMA reference (REF2) and imprinted (MIP2) polymers.

bThe poly-EGDMA reference (REF3) and imprinted (MIP3) polymers. Each polymer was prepared using the same stoichiometries as used for the simulated systems (Supplementary Table S1)
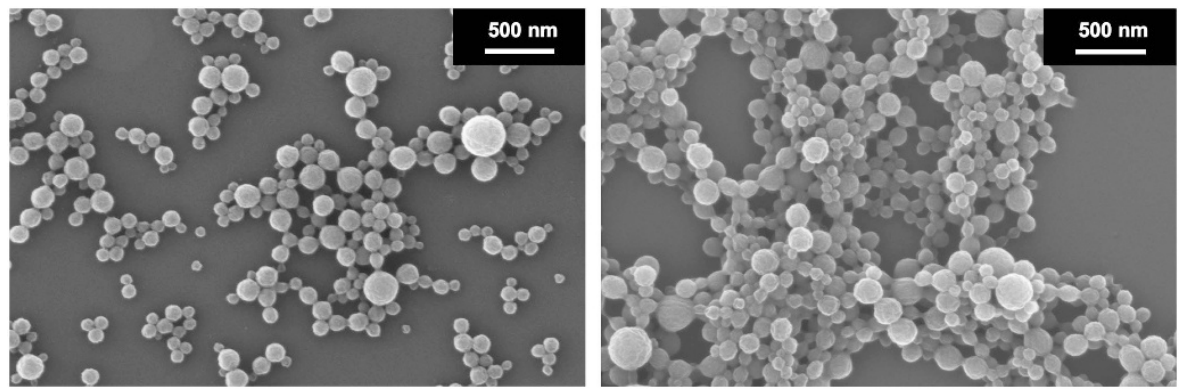

Figure 2 Scanning electron microscope (SEM) micrographs of poly-MAA-co-EGDMA molecularly imprinted polymers (MIPs) (left) and poly-EGDMA reference polymer particles (right). EGDMA, ethylene glycol dimethacrylate; MAA, methacrylic acid. 
phase. The effect would be a decrease in the probability of BPA-MAA interactions in the MIP phase (MD simulations show that both MAA and methacrylate can be solvated in the aqueous phase; Supplementary Figures S13 and S14). This type of event highlights the difficulties associated with predicting partitioning in multicomponent two-phase systems. The weak acidic natures of the phenolic functionalities of the template and of MAA mean that both prefer to engage in interactions with the hydrogen bond-accepting carbonyl oxygens of the abundant EGDMA and even with MAA, rather than with one another (Supplementary Tables S3-S8). However, the combination of a higher concentration of EGDMA with its two carbonyl oxygen atoms leads to a greater incidence of EGDMA-BPA hydrogen bond interactions than MAA-BPA. It is interesting to note that MAA-MAA interactions were not frequent events. Furthermore, the evaluation results (Supplementary Tables S2-S8 and Supplementary Figure S11) indicate that MAA-EGDMA interactions are common and stable, thus leaving even less MAA available for interaction with the template.

Examination of the events at the phase interface demonstrated that BPA and monomers engage in interactions with water. At this position, the polar functionalities are oriented towards the aqueous phase and are saturated with water (limiting association). A slight diffusion of water into the outer layer of the organic phase was observed (Supplementary Figure S2). Accordingly, the formation of imprints complementary in shape and functionality to BPA around the interface would be unlikely because of the solvation of BPA's phenolic moieties by water. The MD simulations of the colloidal prepolymerization mixtures provide insights into the types of interactions that might contribute to the recognition of BPA in the resulting polymer particles, as observed in the BPA-polymer binding studies. Because these studies are performed in aqueous solution, recognition is most likely dependent on a combination of hydrophobic and electrostatic interactions. ${ }^{6}$

To validate the MD data, four polymers were synthesized: BPA-imprinted poly-MAA-co-EGDMA, BPA-imprinted poly-EGDMA and two corresponding reference polymers (see Table 1 and Supplementary Table S1 for compositions). The characterization of their physical properties provided some interesting insights (Table 3 ).

Static light scattering measurements indicated that the poly-EGDMA NPs were 20 times larger than the poly-MAA-co-EGDMA NPs (evaluated using dynamic light scattering) and had a notably broader size distribution. However, scanning electron microscope (SEM) revealed that the poly-EGDMA particles were in the same size range as the poly-MAA-co-EGDMA particles (SEM micrographs in Figure 2). These phenomena were attributed to agglomeration, ${ }^{19,20}$ which was supported by the lower zeta potential of the poly-EGDMA NPs (Table 3) and was attributed to the absence of ionized MAA-derived carboxylates and the lack of electrostatic repulsion between particles to stabilize the dispersion. This effect was evident during synthesis, when particles were observed to agglomerate, as illustrated by the polymer strands connecting beads (Figure 2). The charge surplus at the MIP-water interface contributes to the electrostatic repulsion between suspended colloidal particles. This result correlates well with the MD simulation data, where methacrylate in $\mathrm{m}(\mathrm{DP} 1)$ and $\mathrm{m}(\mathrm{DP} 2)$ is observed either solvated in the aqueous phase or present at the aqueous interface (Supplementary Figures S13 and S14). This result highlighted the need for a stabilizing agent (here SDS) to limit agglomeration when non-charged monomers are used.

Finally, the results from BPA-adsorption/uptake studies (Supplementary Figure S13) show that the poly-EGDMA material bound nearly the same amount of BPA as poly-MAA-co-EGDMA. In addition, no significant difference could be observed in the specific adsorption ( $\left.\mathrm{REF}_{\text {adsorption }}-\mathrm{MIP}_{\text {adsorption }}\right)$ (Supplementary Table S9). Thus, the experimental adsorption results confirmed the theoretical predictions obtained from simulations. MAA is not necessary to create imprinted polymers with affinity for BPA. However, the results from the SEM, static light scattering and dynamic light scattering measurements show that MAA has another role, namely contributing to the stabilization of the emulsion.

In summary, we present the first MD simulations of miniemulsion prepolymerization mixtures and have demonstrated their value in studies of the emulsion-based synthesis of MIP-NPs. Analysis of the simulated emulsions led to the successful prediction of properties related to the final materials, and the prediction showed a strong correlation with the experimental results. The predictive power of MD simulations of these systems was particularly evident through its use as a prognostic tool for the identification of an alternative MIP composition.

\section{EXPERIMENTAL PROCEDURE}

All experimental procedures and detailed summaries of results are presented in the Supporting Information of this communication. Briefly, all-atom, full system MD simulations were performed using the TIP3P water model (the default water model in the simulation software used), with atomic partial charges derived using the ANTECHAMBER software included in the simulation software package AMBER. The general amber force field was used to describe all molecules except water, where the default AMBER force field with adopted TIP3P parameters was used. Periodic boundary conditions were used for bulk representation, and bonds to hydrogen were restrained to allow a longer time step in the performed simulations. A detailed accounting of all experimental procedures, software used and experimental conditions with accompanying citations is presented in the Supporting Information.

\section{CONFLICT OF INTEREST}

The authors declare no conflict of interest.

\section{ACKNOWLEDGEMENTS}

This work was supported by the European Union FP7 WATERMIM (Grant No. 226524), the Linnaeus University, the Swedish Knowledge Foundation (Grant No. 2010-0223) and the Swedish Research Council (Grant No. 2006-6041).

1 Vandenberg, L. N., Hauser, R., Marcus, M., Olea, N. \& Welshons, W. V. Human exposure to bisphenol A (BPA). Reprod. Toxicol. 24, 139-177 (2007).

2 Staples, C., Dorn, P., Klecka, G., O'Block, S. \& Harris, L. A review of the environmental fate, effects, and exposures of bisphenol A. Chemosphere 36, 2149-2173 (1998).

3 Sellergren, B. Molecularly Imprinted Polymers: Man-Made Mimics of Antibodies and their Application in Analytical Chemistry, Vol. 23 (Elsevier, Amsterdam, The Netherlands, 2001).

4 Wulff, G. Molecular imprinting in cross-linked materials with the aid of molecular templates - a way towards artificial antibodies. Angew. Chem. Int. Ed. Engl. 34, 1812-1832 (1995).

5 Whitcombe, M. J., Kirsch, N. \& Nicholls, I. A. Molecular imprinting science and technology: a survey of the literature for the years 2004-2011. J. Mol. Recognit. 27, 297-401 (2014)

6 Dirion, B., Cobb, Z., Schillinger, E., Andersson, L. I. \& Sellergren, B. Water-compatible molecularly imprinted polymers obtained via high-throughput synthesis and experimental design. J. Am. Chem. Soc. 125, 15101-15109 (2003).

7 Karlsson, B. C. G. O'Mahony, J., Karlsson, J. G., Bengtsson, H., Eriksson, L. A. \& Nicholls, I. A. Structure and dynamics of monomer-template complexation: an explanation for molecularly imprinted polymer recognition site heterogeneity. J. Am. Chem. Soc. 131, 13297-13304 (2009).

8 Olsson, G. D., Karlsson, B. C. G., Shoravi, S., Wiklander, J. G. \& Nicholls, I. A Mechanisms underlying molecularly imprinted polymer molecular memory and the role 
of crosslinker: resolving debate on the nature of template recognition in phenylalanine anilide imprinted polymers. J. Mol. Recognit. 25, 69-73 (2012).

$9 \mathrm{Li}, \mathrm{X}$., Li, Y., Dong, C., Jin, P. \& Qi, J. Selective recognition of veterinary drugs residues by artificial antibodies designed using a computational approach. Biomaterials $\mathbf{3 0}$, 3205-3211 (2009)

10 Dong, C., Li, X., Guo, Z. \& Qi, J. Development of a model for the rational design of molecular imprinted polymer: computational approach for combined molecular dynamics/quantum mechanics calculations. Anal. Chim. Acta 647, 117-124 (2009).

11 Vaihinger, D., Landfester, K., Kräuter, I., Brunner, H. \& Tovar, G. E. M. Molecularly imprinted polymer nanospheres as synthetic affinity receptors obtained by miniemulsion polymerisation. Macromol. Chem. Phys. 203, 1965-1973 (2002).

12 Esfandyari-Manesh, M., Javanbakht, M., Dinarvand, R. \& Atyabi, F. Molecularly imprinted nanoparticles prepared by miniemulsion polymerization as selective receptors and new carriers for the sustained release of carbamazepine. J. Mater. Sci. 23, 963-972 (2012).

13 Huang, Y. -P., Liu, Z. -S., Zheng, C. \& Gao, R. -Y. Recent developments of molecularly imprinted polymer in CEC. Electrophoresis 30, 155-162 (2009).

14 Hoshino, Y., Koide, H., Urakami, T., Kanazawa, H., Kodama, T., Oku, N. \& Shea, K. J. Recognition, neutralization, and clearance of target peptides in the bloodstream of living mice by molecularly imprinted polymer nanoparticles: a plastic antibody. J. Am. Chem. Soc. 132, 6644-6645 (2010).
15 Poma, A., Turner, A. P. F. \& Piletsky, S. A. Advances in the manufacture of MIP nanoparticles. Trends Biotechnol. 28, 629-637 (2010).

$16 \mathrm{Xu}$, C., Shen, X. \& Ye, L. Molecularly imprinted magnetic materials prepared from modular and clickable nanoparticles. J. Mater. Chem. 22, 7427-7433 (2012).

17 Yoshimatsu, K., Reimhult, K., Krozer, A., Mosbach, K., Sode, K. \& Ye, L. Uniform molecularly imprinted microspheres and nanoparticles prepared by precipitation polymerization: the control of particle size suitable for different analytical applications. Anal. Chim. Acta 584, 112-121 (2007).

18 Nicholls, I. A., Andersson, H. S. Golker, K. Henschel, H., Karlsson, B. C. G. Olsson, G. D., Rosengren, A. M., Shoravi, S., Suriyanarayanan, S., Wiklander, J. G. \& Wikman, S. Rational design of biomimetic molecularly imprinted materials: theoretical and computational strategies for guiding nanoscale structured polymer development. Anal. Bioanal. Chem. 400, 1771-1786 (2011).

19 Chern, C. S. \& Chen, T. J. Miniemulsion polymerization of styrene stabilized by nonionic surfactant and reactive cosurfactant. Colloid Polym. Sci. 275, 1060-1067 (1997).

20 Hecht, L. L., Wagner, C., Landfester, K. \& Schuchmann, H. P. Surfactant concentration regime in miniemulsion polymerization for the formation of MMA nanodroplets by high-pressure homogenization. Langmuir Acs J. Surf. Colloids 27, 2279-2285 (2011).

Supplementary Information accompanies the paper on Polymer Journal website (http://www.nature.com/pj) 